\title{
THEORY OF NEUTRINO MASSES AND MIXINGS
}

\author{
HITOSHI MURAYAMA \\ Department of Physics, University of California \\ Berkeley, CA 94720, USA \\ Theoretical Physics Group, Lawrence Berkeley National Laboratory \\ University of California, Berkeley, CA 94720, USA \\ E-mail: murayama@lbl.gov
}

\begin{abstract}
Neutrino physics is going through a revolutionary progress. In this talk I review what we have learned and why neutrino mass is so important. Neutrino masses and mixings are already shedding new insight into the origin of flavor. Given the evidences for neutrino mass, leptogenesis is gaining momentum as the origin of cosmic baryon asymmetry. Best of all, we will learn a lot more in the coming years.
\end{abstract}

\section{Introduction}

There is no question that we are in a truly revolutionary moment in neutrino physics. From the previous speakers, Jordap Goodman, Josh Klein 3 Chang-Kee Jung, 3 and Shigeki Aoki, 1 we have seen a wealth of new experimental data in neutrino physics since the previous Lepton Photon conference. We are going through a revolution in our understanding of neutrinos, even in broader context of flavor physics, unification, and cosmology. My job is to go through some of the exciting aspects of these topics.

I found that not only us but also general public is excited about neutrino physics; I've found fortune cookies from SuperK brand on my way from Snowmass meeting to Denver airport at a Chinese restaurant.

We have learned the following important points since the previous Lepton Photon conference:

- Evidence for $\nu_{\mu}$ deficit in atmospheric neutrinos is stronger than ever, with the up/down asymmetry established at more than $10 \sigma$ level in $\nu_{\mu}$-induced events. $1 \mathrm{We}$ are more than $99 \%$ certain $\nu_{\mu}$ are converted mostly to $\nu_{\tau}$. Current K2K data support this evidence. 3

- Putting SuperKamiokande and SNO

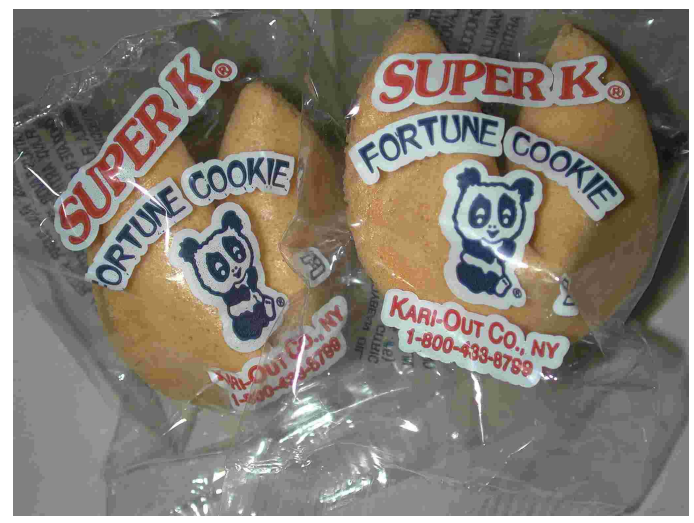

Figure 1. Fortune cookies from SuperK brand I found in Colorado.

data together, we are certain at $3 \sigma$ level that the solar $\nu_{e}$ must have converted to $\nu_{\mu}$ or $\nu_{\tau}$.

- Such neutrino conversions are most likely due to neutrino oscillations. Other possibilities, such as neutrino decay, violation of equivalence principle, spinresonant rotation, exotic flavor-changing interactions, are still possible, but are either squeezed phenomenologically or rely on models that are theoretically not motivated or esthetically not pleasing.

- Tiny neutrinos masses required in neutrino oscillation for atmospheric and so- 
lar neutrinos are the first evidence for the incompleteness of the Minimal Standard Model.! Even apart from oscillations, any explanation to these phenomena require physics beyond the Standard Model.

Given this dramatic progress in experimental results, it is interesting to see how good insight theorists had had on neutrino physics. Here is the list of typical views among theorists on neutrino physics back in 1990:

- Solar neutrino problem must be solved by the small angle MSW solution because it is so cute.

- The natural scale for $\nu_{\mu}-\nu_{\tau}$ oscillation is $\Delta m^{2}=10-100 \mathrm{eV}^{2}$ because it is cosmologically interesting.

- The mixing angle for $\nu_{\mu}-\nu_{\tau}$ oscillation is of the order of $V_{c b}$.

- Atmospheric neutrino anomaly must go away because it requires an ugly large angle, not suggested by simple grandunified theories.

Looking back at this list, the first one is most likely wrong as we will see later, and (2-4) are all wrong. You can see that theorists had had great insight into the nature of neutrinos.

The rest of the talk is organized in the following manner. I first review the situation with the global fit to solar neutrino data after SNO results came out. Then models of neutrino masses and mixings, in the broader context of models of flavor, are briefly reviewed. After that, the idea of leptogenesis is explained, which is one of the main ideas now to explain the cosmological matter anti-matter asymmetry. Finally I will discuss what we can look forward to in the near future.

\section{Global Fits}

I review the global fits to the solar neutrino data including SNO. But before doing so, I spend some time discussing the parameter space of two-flavor neutrino oscillation.

Traditionally, neutrino oscillation data had been shown on the $\left(\sin ^{2} 2 \theta, \Delta m^{2}\right)$ plane. This parameterization, however, covers only a half of the parameter space. We instead need to use, for example, $\left(\tan ^{2} \theta, \Delta m^{2}\right)$ to present data. This point had been recognized for a long time in the context of three-flavor mixing 5 but two-flavor analyses had always been presented on the $\left(\sin ^{2} 2 \theta, \Delta m^{2}\right)$ except as a limit of three-flavor analyses. Because you will see plots that cover the full parameter space soon and also in the future, I will briefly explain this point.

The oscillation occurs because the flavor eigenstate (i.e. $S U(2)_{L}$ partner of charged leptons) and the mass eigenstates are not the same. Let us talk about $\nu_{e}$ and $\nu_{\mu}$ for the sake of discussion. The mass eigenstates are then given by two orthogonal linear combination of them:

$$
\begin{aligned}
& \nu_{1}=\nu_{e} \cos \theta+\nu_{\mu} \sin \theta, \\
& \nu_{2}=-\nu_{e} \sin \theta+\nu_{\mu} \cos \theta
\end{aligned}
$$

As a convention, we can always choose $\nu_{2}$ to be heavier than $\nu_{1}$ without a loss of generality. Now the question is how much we should vary $\theta$. If you use $\sin ^{2} 2 \theta \in[0,1]$ as your parameter, $\theta$ can go from 0 to $45^{\circ}$ to exhaust all possibilities of $\sin ^{2} 2 \theta$. However, for $\theta \leq 45^{\circ}$, $\nu_{1}$ always contains more $\nu_{e}$ than $\nu_{\mu}$. To represent the possibility of $\nu_{1}$ dominated by $\nu_{\mu}$ rather than $\nu_{e}$, we need to allow $\theta$ to go beyond $45^{\circ}$ up to $90^{\circ}$. Then $\sin ^{2} 2 \theta$ folds over at 1 and comes back down to 0 . Clearly, $\sin ^{2} 2 \theta$ is not the right parameter for global fits to the data.

When the oscillation is purely that in the vacuum (no matter effect), the oscillation 
probability depends only on $\sin ^{2} 2 \theta$,

$$
P\left(\nu_{e} \rightarrow \nu_{\mu}\right)=\sin ^{2} 2 \theta \sin ^{2} 1.27 \frac{\Delta m^{2}}{E} L,
$$

with $\Delta m^{2}$ in $\mathrm{eV}^{2}, E$ in $\mathrm{GeV}$ and $L$ in $\mathrm{km}$. Therefore, it is the same for $\theta$ and $90^{\circ}-\theta$, explaining why $\sin ^{2} 2 \theta$ had been used for fits in the past. In the presence of the matter effect, however, the oscillation probability is different for $\theta$ and $90^{\circ}-\theta$.

To cover all physically distinct possibilities, one possible parameter choice is $\sin ^{2} \theta$, that ranges from 0 to 1 for $\theta \in\left[0^{\circ}, 90^{\circ}\right]$, and shows the symmetry under $\theta \leftrightarrow 90^{\circ}-\theta$ in the absence of the matter effect as a reflection with respect to the axis $\theta=45^{\circ}$ on a linear scale. This is a perfectly adequate choice for representing atmospheric neutrino data, for instance when matter effect is included in $\nu_{\mu} \leftrightarrow \nu_{s}$ oscillation. On a log-scale, however, $\tan ^{2} \theta$ shows the symmetry manifestly as $\theta \rightarrow$ $90^{\circ}-\theta$ takes $\tan ^{2} \theta \rightarrow \cot ^{2} \theta=1 / \tan ^{2} \theta$. Especially for fits to the solar neutrino data, we need to cover a wide range of mixing angles, and hence a log-scale; that makes $\tan ^{2} \theta$ essentially the unique choice for graphically presenting the fits. The range of mixing angle $0^{\circ} \leq \theta \leq 45^{\circ}$ is what had been covered traditionally with the parameter $\sin ^{2} 2 \theta$, and we call it "the light side," while the remaining range $45^{\circ} \leq \theta \leq 90^{\circ}$ "the dark side" because it had been usually neglected in the fits. 0

Now that SNO charged-current data is available, we would like to see its impact on the global fit to the solar neutrino data. Of course, the most important lesson from the SNO data is that there is an additional active neutrino component $\nu_{a}$ (i.e., a linear combination of $\nu_{\mu}$ and $\nu_{\tau}$ ) coming from the Sun. The next quantitative lesson is that BP00 flux calculation of ${ }^{8} \mathrm{~B}$ neutrino flux is verified within its error, as discussed by Josh Klein in his talk. 目

I show results from the global fits. The first one is a two-flavor fits for $\nu_{e}$ to $\nu_{a}$ oscillation, using BP2000 solar neutrino flux calculations in Fig. 2. Four regions of the param-

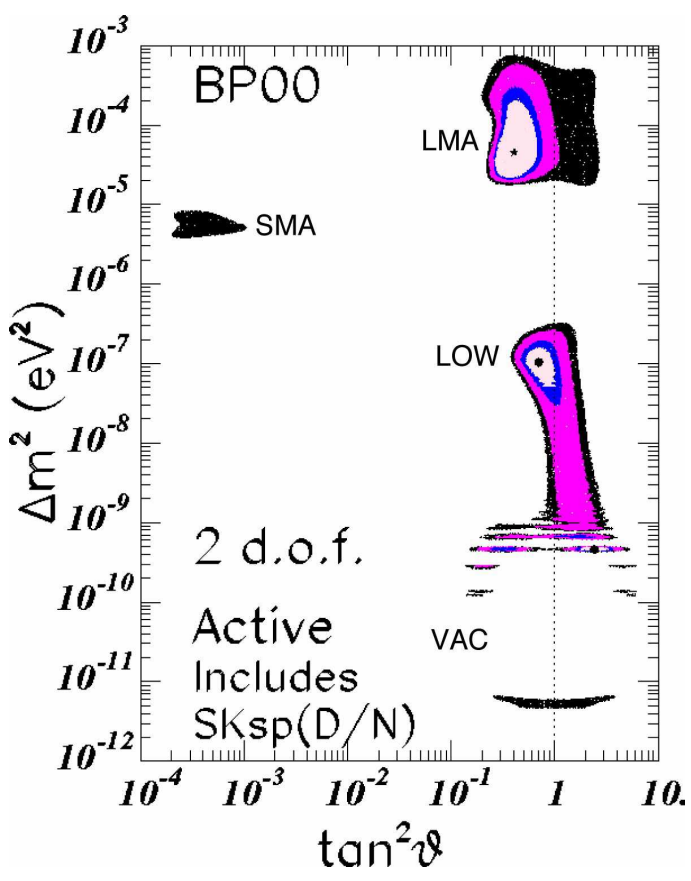

Figure 2. Two-flevor fit to solar neutrino data for $\nu_{e}$ to $\nu_{a}$ oscillationt. The confidence levels are $90 \%$, $95 \%, 99 \%$, and $99.73 \%(3 \sigma)$.

eter space, LMA (Large Mixing Angle MSW solution), SMA (Small Mixing Angle MSW solution), LOW (MSW solution with LOW $\Delta m^{2}$ ), and VAC (VACuum oscillation solution), that fit the data can be seen. LMA solution is the best fit to the current data.

One of the concerns in solar neutrino fits has been that been that solar ${ }^{8} \mathrm{~B}$ neutrino flux is very sensitive to solar parameters. Thanks to the SNO data, we can now drop the predicted flux entirely from the fit and obtain equally good fit, as shown in Fig. 3 .

To account for both solar and atmospheric neutrino oscillations, we need threeflavor analyses. The standard parameterization of the mixing among neutrinos, MNS

\footnotetext{
${ }^{a}$ This concern has been greatly ameliorated by the agreement of helioseignology data and the standard solar model, however.
} 


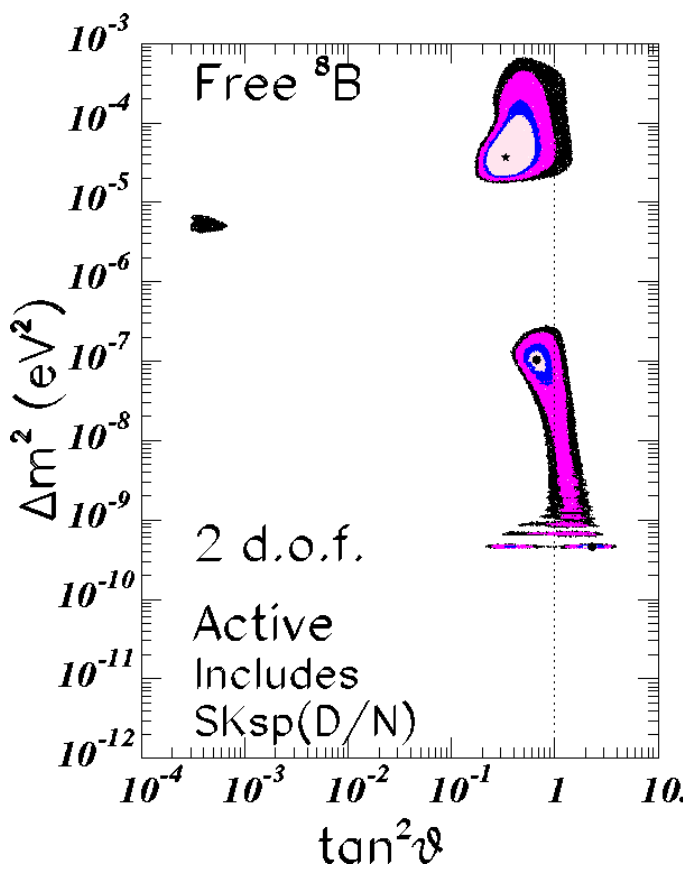

Figure 3. Two-flavor fit taking the solar ${ }^{8} \mathrm{~B}$ neutrino flux as a free parameter

(Maki-Nakagawa-Sakata) matrix, is given by

$$
\begin{aligned}
& U_{M N S}=\left(\begin{array}{ccc}
U_{e 1} & U_{e 2} & U_{e 3} \\
U_{\mu 1} & U_{\mu 2} & U_{\mu 3} \\
U_{\tau 1} & U_{\tau 2} & U_{\tau 3}
\end{array}\right) \\
& =\left(\begin{array}{ccc}
1 & 0 & 0 \\
0 & c_{23} & s_{23} \\
0 & -s_{23} & c_{23}
\end{array}\right)\left(\begin{array}{ccc}
c_{13} & 0 & s_{13} e^{-i \delta} \\
0 & 1 & 0 \\
-s_{13} e^{i \delta} & 0 & c_{13}
\end{array}\right) \\
& \quad \times\left(\begin{array}{ccc}
c_{12} & s_{12} & 0 \\
-s_{12} & c_{12} & 0 \\
0 & 0 & 1
\end{array}\right)
\end{aligned}
$$

The angle $\theta_{13}$ is currently undetermined, except that there is an upper bound $\sin \theta_{13} \lesssim$ 0.16 from reactor neutrino experiments $\$ 10$ for $\Delta m^{2}=3 \times 10^{-3} \mathrm{eV}^{2}$ preferred by the atmospheric neutrino data. Fortunately, the smallness of $\theta_{13}$ essentially decouples solar and atmospheric neutrino oscillation, allowing us to interpret data with separate twoflavor fits. The convention here is that the mass eigenstates $\nu_{1}$ and $\nu_{2}$ have small mass splitting for the solar neutrino oscillation $\Delta m_{12}^{2} \lesssim 2 \times 10^{-4} \mathrm{eV}^{2}$, while $\nu_{2}$ and $\nu_{3}$ have

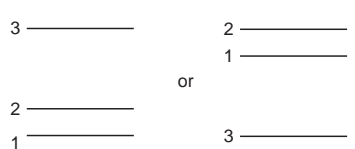

Figure 4. The mass spectrum of three neutrino mass eigenstates.

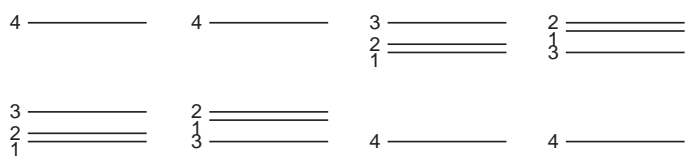

Figure 5. The $3+1$ mass spectra of four neutrino mass eigenstates.

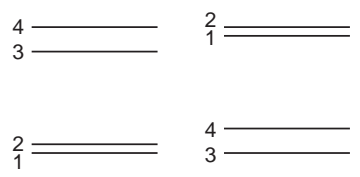

Figure 6 . The $2+2$ mass spectra of four neutrino mass eigenstates.

the splitting $\Delta m_{23}^{2} \simeq 3 \times 10^{-3} \mathrm{eV}^{2}$, as shown in Fig. \&. I've put $\nu_{2}$ above $\nu_{1}$ assuming we are on the "light side" of solar neutrino oscillation. The "dark side" of solar neutrino oscillation would correspond to the opposite order. Future improvements in the solar neutrino data would allows us to discriminate between the two. In addition, we still do not know if $\nu_{3}$ should be above or below the solar doublet. The remaining issues are (1) to determine the solar neutrino oscillation parameters, (2) the ordering of mass eigenstates, and (3) $\theta_{13}$.

When LSND oscillation signal (see talk by Aoki 4 ), that prefers $\Delta m^{2} \sim \mathrm{eV}^{2}$, is also considered, we have to accommodate three different orders of magnitude of $\Delta m^{2}$ values and hence four mass eigenstates. Because of three $\Delta m^{2}$, the mass spectrum now has $3 !=$ 6 possibilities shown in Figs. 司 and 6 .

Now that both solar and atmospheric neutrino data disfavor oscillations into pure sterile state, the scenario with sterile neutrino is getting squeezed. For example, the comparison of sterile and active case can be 


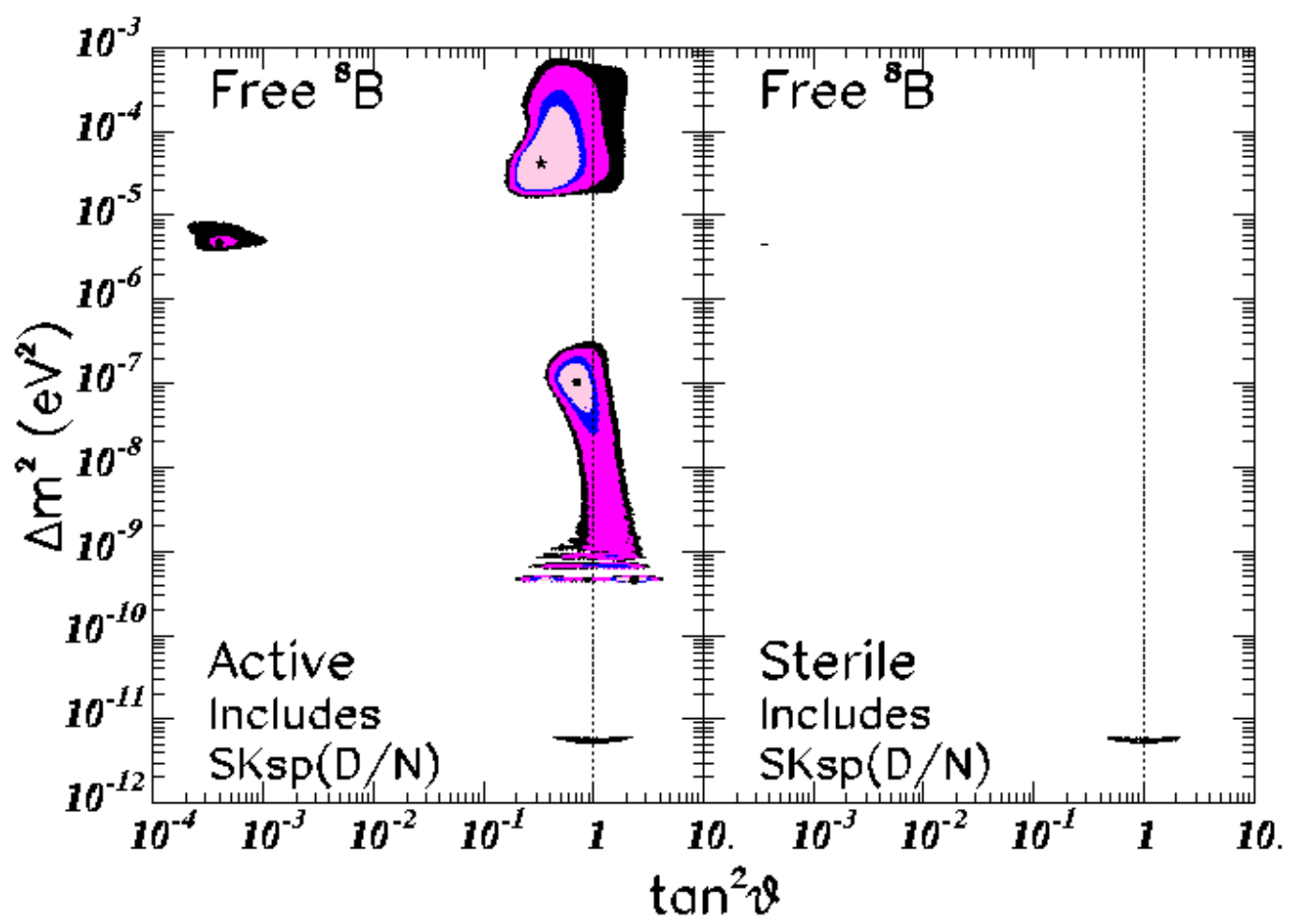

Figure 7. A global fit interpolating the pure active and pure sterile cases. 0 See text for the precautions on the definition of confidence levels that differ from Figs. 6 and 3 .

done by interpolating the two cases with an additional parameter $\eta$. The state that solar $\nu_{e}$ oscillates to is a mixture of an active and sterile neutrino, $\nu_{a} \cos \eta+\nu_{s} \sin \eta$. Then one can fit the data with three degrees of freedom, $\Delta m^{2}, \tan ^{2} \theta$, and $\eta$, and finds that the fit is better for $\eta=0$ (pure active case). A word of caution here is that the definition of the confidence levels is now looser, making the allowed regions bigger. The confidence levels $90 \%, 95 \%, 99 \%$, and $99.73 \%$ in Fig. 五 would correspond to $96 \%, 98 \%, 99.7 \%$, and $99.92 \%$ for pure two-flavor case. Nonetheless, it is clear that the pure sterile case provides a much less good fit.

However, the phenomenological motivation for having a sterile state comes from the combination of LSND, atmospheric, and solar neutrino data, and it requires full fourflavor analysis. In the case of $3+1$ spec- tra, the mixing angle for LSND oscillation is related to the MNS matrix elements as $\sin ^{2} 2 \theta_{\mathrm{LSND}}=4\left|U_{e 4}\right|^{2}\left|U_{\mu 4}\right|^{2} \gtrsim 0.01$. On the other hand, $\left|U_{\mu 4}\right|$ cannot be too large because of CDHS data and atmospheric neutrino data, while $\left|U_{e 4}\right|^{2}$ either because of Bugey reactor neutrino data. 11 This type of spectra is only marginally allowed 12 , and fits prefer $2+2$ spectra 13 However, $2+2$ spectra are also getting squeezed now that both solar and atmospheric neutrino data disfavor oscillations into pure sterile state. If solar neutrino oscillation is into a pure active state, the atmospheric neutrino oscillation must be into a pure sterile state, and vice versa. The way this type of spectra can fit the data is to find a compromise between the requirements of sufficient active component in both oscillations. Detailed numerical analysis showed that such a compromise is still possible and 


\section{fermion masses}

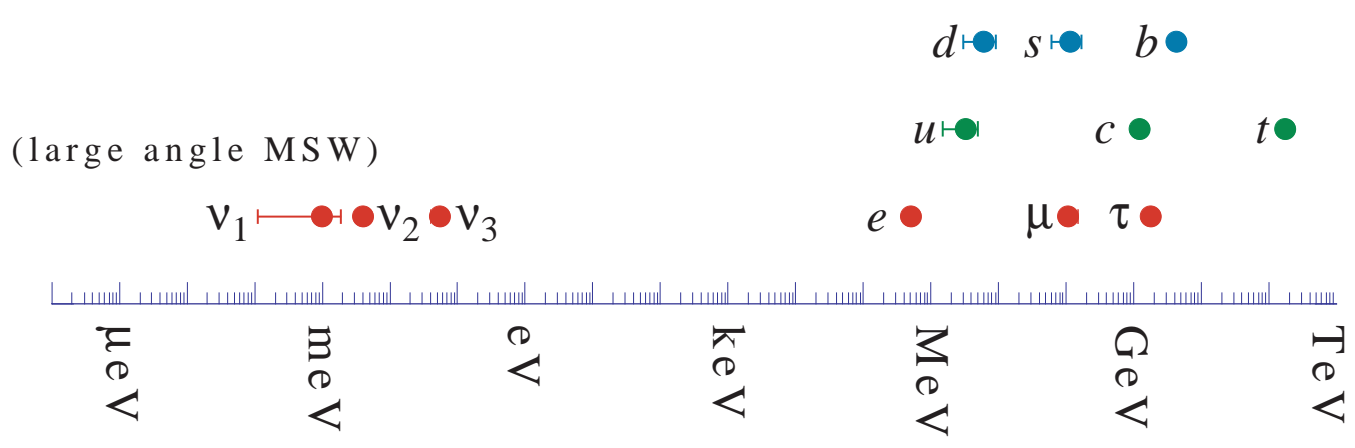

Figure 8. Mass spectrum of quarks and leptons. LMA solution to the solar neutrino problem is assumed, while the range given for $\nu_{1}$ mass is basically just a guess.

the sterile neutrino is still allowed. $\mathrm{l}$ The final verdict will be given by Mini-BooNE as we will see later.p

\section{Models}

Now that neutrinos appear massive, despite what Standard Model has assumed for decades, we need to somehow incorporate the neutrino masses into our theory. The most striking facts about the neutrino sector are (1) the masses are very small, and (2) mixing angles appear large. Looking at the mass spectrum of quarks and leptons, it is especially bizarre that even the third-generation mass is so low compared to quarks and charged leptons. An explanation is clearly called for.

The minute you talk about masses of spin $1 / 2$ particles, you need both spin up and down states because you can stop any massive particle. When the particle is at a relativistic speed, a more useful label is leftor right-handed states. For strictly mass-

\footnotetext{
${ }^{b}$ There is an intriguing possibility that all three oscillation data can be explained without a sterile neutrino, if there is $\mathrm{CPT}$ violation that allows differe mass spectra between neutrinos and anti-neutrinos. 14 This possibility can be tested by having anti-neutrino run at Mini-BooNE beyond the planned neutrino run.
}

less particles, left- and right-handed states are completely independent from each other and you do not need both of them; this is how neutrinos are described in the Standard Model. Once they are massive, though, we need both, so that we can write a mass term using both of them:

$$
\mathcal{L}_{\text {mass }}=m_{D}\left(\overline{\nu_{L}} \nu_{R}+\overline{\nu_{R}} \nu_{L}\right) .
$$

But then the mass term is exactly the same as the other quarks and leptons, and why are neutrinos so much lighter?

The so-called seesaw mechanism 15 is probably the most motivated explanation to the smallness of neutrino masses. The first step is to rewrite the mass term Eq. (5) in a matrix form

$$
\mathcal{L}_{\text {mass }}=\frac{1}{2}\left(\nu_{L} \overline{\nu_{R}}\right)\left(\begin{array}{cc}
0 & m_{D} \\
m_{D} & 0
\end{array}\right)\left(\begin{array}{l}
\nu_{L} \\
\overline{\nu_{R}}
\end{array}\right)+c . c .
$$

Here, I had to put $\nu_{L}$ and $\overline{\nu_{R}}$ (CP conjugate of $\nu_{R}$ ) together so that both of them are left-handed and are allowed to be in the same multiplet. The problem was that we (at least naively) expect the "Dirac mass" $m_{D}$ to be of the same order of magnitudes as other quarks and lepton masses in the same gener-

\footnotetext{
$\overline{{ }^{c} \text { Recently, alternati }}$ explanation using extra dimensions had appeared.16
} 
ation which would be way too large (Fig. 9). The point is that the right-handed neutrino is completely neutral under the standard-model gauge groups and is not tied to the electroweak symmetry breaking $(v=246 \mathrm{GeV})$ to acquire a mass. Therefore, it can have a mass much larger than the electroweak scale without violating gauge invariance, and the mass term is (Fig. 10)

$$
\mathcal{L}_{\text {mass }}=\frac{1}{2}\left(\nu_{L} \overline{\nu_{R}}\right)\left(\begin{array}{cc}
0 & m_{D} \\
m_{D} & M
\end{array}\right)\left(\begin{array}{l}
\nu_{L} \\
\overline{\nu_{R}}
\end{array}\right)+c . c . .
$$

Because one of the mass eigenvalues is clearly dominated by $M \gg m_{D}$, while the determinant is $-m_{D}^{2}$, the other eigenvalue must be suppressed, $-m_{D}^{2} / M \ll m_{D}$ (Fig. 11). This way, physics at high-energy scale $M$ suppresses the neutrino mass in a natural way. In order to obtain the mass scale for the atmospheric neutrino oscillation $\left(\Delta m_{\mathrm{atm}}^{2}\right)^{1 / 2} \sim$ $0.05 \mathrm{eV}$, and taking the third generation mass $m_{D} \sim m_{t} \sim 170 \mathrm{GeV}$, we find $M=$ $m_{D}^{2} / m_{\nu} \sim 0.6 \times 10^{15} \mathrm{GeV}$. It is almost the grand-unification scale $2 \times 10^{16} \mathrm{GeV}$ where all gauge coupling constants appear to unify in th minimal supersymmetric standard model.

Indeed, the seesaw mechanism was motivated by $S O(10)$ grand-unified models which include right-handed neutrinos automatically together with all other quarks and leptons in irreducible 16-dimensional multiplets. $S O(10)$ GUT also has an esthetic appeal, being the smallest anomaly-free gauge group with chiral fermions, while not requiring additional fermions beyond the right-handed neutrinos. But it has a slight problem: it is too predictive. The simplest version of the model predicts $m_{c}=m_{s}=m_{\mu}$ at the GUTscale and no CKM (Cabibbo-KobayashiMaskawa) mixing. The art of unified model building is how to break the naive prediction to a realistic one.

There are many many models, with or without grand unification, of neutrino masses and mixings. There are papers submitted by C.S. Lam, Alexandre Khodin, Joe Sato,

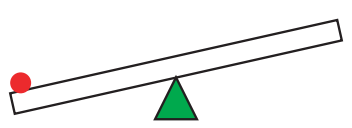

Figure 9. Too large Dirac mass of neutrinos.

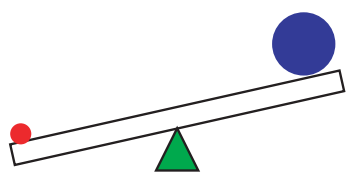

Figure 10. We can put a large mass to the righthanded neutrino without violating gauge invariance.

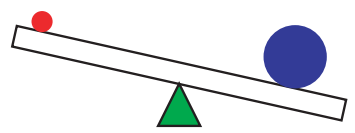

Figure 11. Then the mass of the neutrino becomes light.

Koichi Matsuda, Bruce McKellar, and Carl H. Albright to this conference. The bottomline is that one can construct nice unified models of quark and lepton masses, especially within $S U(5)$ or $S O(10)$ unification. I do not intend to go into an exhaustive review of proposed models because I can't di Instead, I'd like to discuss how we might eventually arrive at understanding masses and mixings in a bottom-up approach.

Looking at quarks, the masses are very hierarchical, and mixings are small. The masses of charged leptons are also very hierarchical. Even though we are used to hierarchical mass spectrum, it is actually quite bizarre: why do particles with exactly the same quantum numbers have such different masses, and mix little? In graduate quantum mechanics, didn't we learn that states with same quantum numbers have typically similar energy levels and mix substantially? A very naive answer to the puzzle is that different generations of quarks presumably have

$\overline{{ }^{d} \text { I hope no list angers }}$ less people than an incomplete list. 
different hidden quantum numbers we have not identified yet. We call them "flavor quantum numbers." Flavor quantum numbers distinguish different generations, allowing them to have very different energy levels (masses) and forbidding them to mix substantially.

First question we should ask then is if there is need for fundamental distinction among three neutrinos. If you look at the currently-favored LMA solution to the solar neutrino problem, two mass-squared differences are not that different, $\Delta m_{\mathrm{atm}}^{2} \simeq$ $1-7 \times 10^{-3} \mathrm{eV}^{2}, \Delta m_{\mathrm{LMA}}^{2} \simeq 0.2-6 \times 10^{-4} \mathrm{eV}^{2}$ (both at $99 \% \mathrm{CL}$ ). The mixing angles $\theta_{12}$, $\theta_{23}$ are both large. Even though $\theta_{13}$ is usually said to be small, $\left|U_{e 3}\right|=\sin \theta_{13} \lesssim 0.16$ at $\Delta m_{\text {atm }}^{2}=3 \times 10^{-3} \mathrm{eV}^{2}$, it is smaller than $\left|U_{\mu 3}\right| \simeq\left|U_{\tau 3}\right| \simeq 1 / \sqrt{2}=0.71$ only by a factor of 2.3. Furthermore at the low end of $\Delta m_{\text {atm }}^{2}, \sin \theta_{13}$ may be still sizable $(0.2(0.4)$ at $\left.\Delta m_{\mathrm{atm}}^{2}=2(1) \times 10^{-3} \mathrm{eV}^{2}\right)$. It is not clear yet if $\theta_{13}$ is so small. If there is no hierarchy and mixing is large, we apparently do not need new quantum numbers to distinguish three generation of neutrinos.

But isn't near-maximal mixing suggested by atmospheric neutrino data special? Isn't there a special reason for it? If there is no distinction among three generations of neutrinos, isn't even a small hierarchy between atmospheric and solar mass-squared differences puzzling? To address these questions, we ran Monte Carlo over seesaw mass matrices 17 It turns out that the distribution is peaked at $\sin ^{2} 2 \theta=1$ for atmospheric neutrino mixing, and the ratio of two masses-squared differences $\Delta m_{\text {solar }}^{2} / \Delta m_{\text {atm }}^{2} \sim 0.1$ is the most likely value. The lesson here is that, if there is no fundamental distinction among three generations of neutrinos, the apparent pattern of masses and mixings comes out quite naturally. Of course, "randomness" behind Monte Carlo is just a measure of our ignorance. But complicated unknown dynamics of flavor at some high-energy scale may well appear to produce random numbers in the low-energy

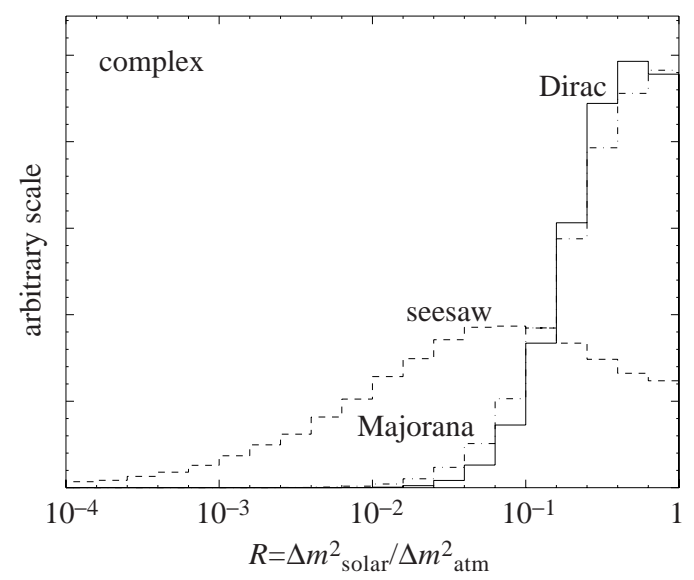

Figure 12. The ratio of two mass-squared differences in randomly generated $3 \times 3$ neutrino mass matrices 18 For the seesaw case, the peak is around $\Delta m_{\text {solar }}^{2} / \Delta m_{\text {atm }}^{2} \sim 0.1$.

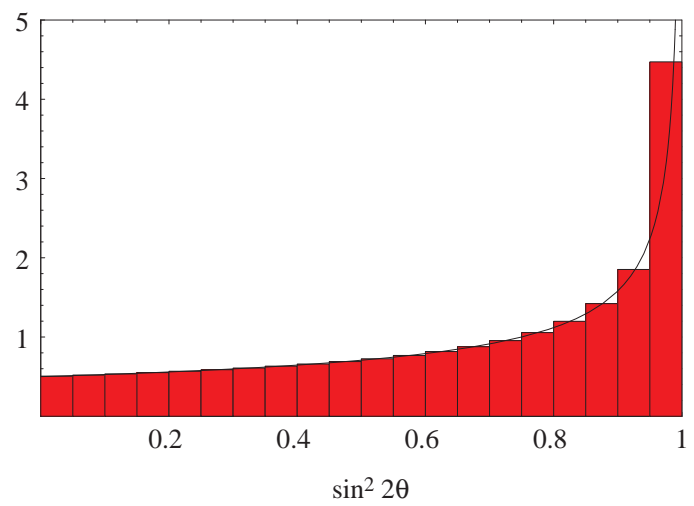

Figure 13. $\sin ^{2} 2 \theta_{23}$ in randemly generated $3 \times 3$ neutrino seesaw mass matrices. 18

theory. We call such a situation "anarchy." And the peak in the mixing angle distribution can be understood in terms of simple group theory 18 In fact, the anarchy predicts that all three mixings angles are peaked in $\sin ^{2} 2 \theta$ distributions at maximum. This does not sound quite right for $\sin ^{2} 2 \theta_{13}$, but we find that three out of four distributions, $\theta_{12}$, $\theta_{23}, \Delta m_{\text {solar }}^{2} / \Delta m_{\text {atm }}^{2}$, prefer what data suggest. I find it quite reasonable. If you take this idea seriously, then $\sin ^{2} 2 \theta_{13}$ must be basically just below the current limit and we hope to see it sometime soon.

What about quarks and charged leptons? 
Here we clearly see a need for fundamental distinction among three generations. are definitely not anarchical; they needed ordered hierarchical structure. Let us suppose the difference among three generations is just a new charge, namely a flavor $U(1)$ quantum number. As a simple exercise, we can assign the following flavor charges consistent with $S U(5)$-type unification:

$$
\begin{aligned}
& \mathbf{1 0}(Q, \bar{U}, \bar{E})(+2,+1,0), \\
& \mathbf{5}^{*}(L, \bar{D})(+1,+1,+1) .
\end{aligned}
$$

All three generations of $L$ have the same charge because of anarchy: no fundamental distinction among them. As we saw, neutrino masses and mixings come out reasonably well from this charge assignment. With $S U(5)$ like unification, right-handed down quarks, that belong to the same $5^{*}$ multiplets with left-handed lepton doublets, also have the same charge for all three generations. It is intriguing that large mixing among righthanded quarks is consistent with what we know about quarks, because the CKM matrix is sensitive only to particles that participate in charged-current weak interaction, namely left-handed quarks. Even if right-handed quarks are maximally mixed, we wouldn't know. On the other hand, 10 multiplets, that contain left-handed quark doublets, righthanded up quarks and right-handed leptons, need differentiation among three generations. Here we assigned the charges +2 for the first generation, +1 for the second, and no charge for the third. This way, top quark Yukawa coupling is allowed by the flavor charges, but all other Yukawa couplings are forbidden. Now suppose the flavor charge is broken by a small breaking parameter $\epsilon \sim 0.04$ that carries charge -1 . Then all other entries of Yukawa matrices are now allowed but regulated by powers of the small parameter $\epsilon$. Then we find that the ratio of quarks and

${ }^{e}$ See also Riccardo Barbieri at this conference. 19 lepton masses are

$$
\begin{aligned}
& m_{u}: m_{c}: m_{t} \sim m_{d}^{2}: m_{s}^{2}: m_{b}^{2} \\
& \sim m_{e}^{2}: m_{\mu}^{2}: m_{\tau}^{2} \sim \epsilon^{4}: \epsilon^{2}: 1 .
\end{aligned}
$$

Namely, the up quarks are doubly hierarchical than down quarks and charged leptons, consistent with what we see.

I'd like to emphasize that the "anarchy" is actually a peaceful ideology, nothing radical. According to Merriam-Webster dictionary, anarchy is defined as "A utopian society of individuals who enjoy complete freedom without government." I'm just saying that neutrinos work peacefully together to freely mix and abolish hierarchy, without being forced by any particular structure. It predicts LMA solution to the solar neutrino problem, $\sin ^{2} 2 \theta_{13}$ must be just below the current limit, and $O(1) \mathrm{CP}$ violating phase. This is an ideal scenario for very long-baseline neutrino oscillation experiments, requiring many countries to be involved. It is therefore proglobalization!

We have seen how new flavor quantum numbers can determine the structure of masses and mixings among quarks and leptons. Theorists of course argue about what the correct charge assignment is. How we will know if any of such flavor quantum numbers are actually right? It will be a long-shot program, needing many new data such as $\sin ^{2} 2 \theta_{13}$, solar neutrinos, possible $\mathrm{CP}$ violation in the neutrino sector as well as more details in the quark sector and even charged leptons (EDM), B-physics, Lepton Flavor Violation, even proton decay. Because the difference in flavor quantum numbers suppress flavor mixing, the pattern of flavor violation must be consistent with assigned flavor quantum numbers. Details of these flavor-violating phenomena could eventually tell us what new quantum number assignment is correct. It is not clear if the origin of flavor is at the energy scale accessible by any accelerator experiment; it may be up at the unification scale. However, we may still 
learn enough information to be able to reconstruct a plausible theory of the origin of flavor, masses, and mixings. I call such a program "archaeology" in the best sense of the word. For example, we can never recreate the conditions of ancient world. But by studying fossils, relics, ruins, and employing multiple techniques, we can come up with satisfactory plausible theories of what had happened. Cosmology is by nature an archaeology. You can't recreate Big Bang. But cosmic microwave background is a wonderfully colorful beautiful dinosaur that tells us great deal about the history of Universe. In the same sense, a wealth of flavor data can point us to the correct theory.

For this purpose, Lepton Flavor Violation (LFV) is a crucial subject. Now that neutrinos appear to oscillate, i.e., convert from one flavor to another, there must be corresponding process among charged leptons as well. Unfortunately neutrino masses themselves do not lead to sizable rates of LFV processes. However, many extensions of physics beyond the Standard Model, most notably supersymmetry, 20 tend to give LFV processes at the interesting levels, such as $\mu \rightarrow e \gamma$, $\mu \rightarrow e$ conversion, $\tau \rightarrow \mu \gamma$, etc. The violation of overall lepton numbers, such as neutrinoless double beta decay, would be also extremely important.f]

\section{Leptogenesis}

One of the primary interest in flavor physics is the origin of the cosmic baryon asymmetry. From the Big-Bang Nucleosynthesis, we know that there is only a tiny asymmetry in the baryon number, $n_{B} / n_{\gamma} \approx 5 \times 10^{-10}$. In other words, there was only one excess quark out of ten billion that survived the annihilation with anti-quarks. Leptogenesis is a possible origin of such a small asymmetry using

$\overline{f_{\text {See also John Ellis }}}$ at this conference, 21 and papers submitted by Funchal Renata Zukanovich, and Bueno et al to this conference. neutrino physics, a possibility that is gaining popularity now that we seem to see strong evidence for neutrino mass.

The original baryogenesis theories used grand-unified theories, 22 because grand unification necessarily breaks the baryon number. Suppose a GUT-scale particle $X$ decays out of equilibrium with direct $\mathrm{CP}$ violation $B(X \rightarrow q) \neq B(\bar{X} \rightarrow \bar{q})$. Then it can create net baryon number $B \neq 0$ in the final state from the initial state of no baryon number $B=0$. It is interesting that such a direct $\mathrm{CP}$ violation indeed had been established in neutral kaon system in this conference (see R. Kessler23 and L. IconomidouFayard24 in this proceedings). However, the original models preserved $B-L$ and hence did not create net $B-L$, that turned out be a problem.

The Standard Model actually violates $B .25$ In the Early Universe when the temperature was above $250 \mathrm{GeV}$, there was no Higgs boson condensate and $W$ and $Z$ bosons were massless (so where all quarks and leptons). Therefore $W$ and $Z$ fields were just like electromagnetic field in the hot plasma and were fluctuating thermally. The quarks and leptons move around under the fluctuating $W$ field background. To see what they do, we solve the Dirac equation for fermions coupled to $W$. There are positive energy states that are left vacant, and negative energy states that are filled in the "vacuum." As the $W$ field fluctuates, the energy levels fluctuate up and down accordingly. Once in a while, however, the fluctuation becomes so large that all energy levels are shifted by one unit. Then you see that one of the positive energy states is now occupied. There is now a particle! This process occurs in the exactly the same manner for every particle species that couple to $W$, namely for all left-handed lepton and quark doublets. This effect is called the electroweak anomaly. Therefore the electroweak anomaly changes (per generation) $\Delta L=1$, and $\Delta q=1$ for all three colors, and hence 


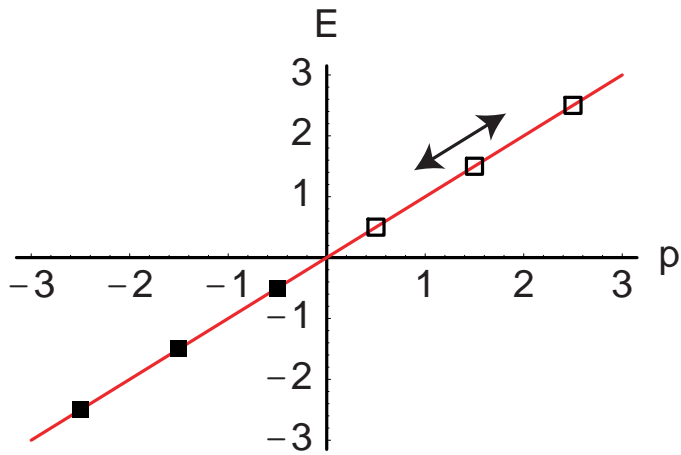

Figure 14. The energy levels of the Dirac equation in the presence of fluctuating $W$-field move up and down. All negative energy states are occupied while the positive energy states vacant in the "vacuum" configuration.

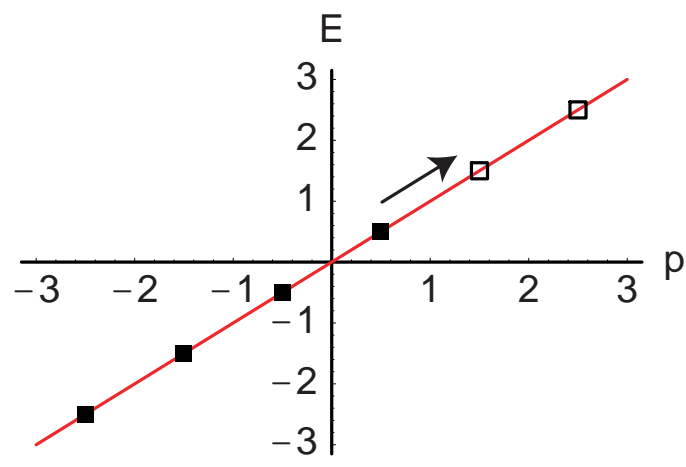

Figure 15. Once in a while, the fluctuation in the $W$ field becomes so large that the energy levels of the Dirac equation in the presence of fluctuating $W$-field shift all the way by one unit. Then a positive energy state is occupied and a particle is created.

$\Delta B=1$. Note that $\Delta(B-L)=0$; the electroweak anomaly preserves $B-L$.

Because of this process, the pre-existing $B$ and $L$ are converted to each other to find the chemical equilibrium at $B \sim 0.35(B-L)$, $L \sim-0.65(B-L)$.27 In particular, even if there was both $B$ and $L$, both of them get washed out if $B-L$ was zero.

Given this problem, there are now two major directions in the baryogenesis. One is the electroweak baryogenesis 26 where you try to generate $B=L$ at the time of the electroweak phase transition so that they do

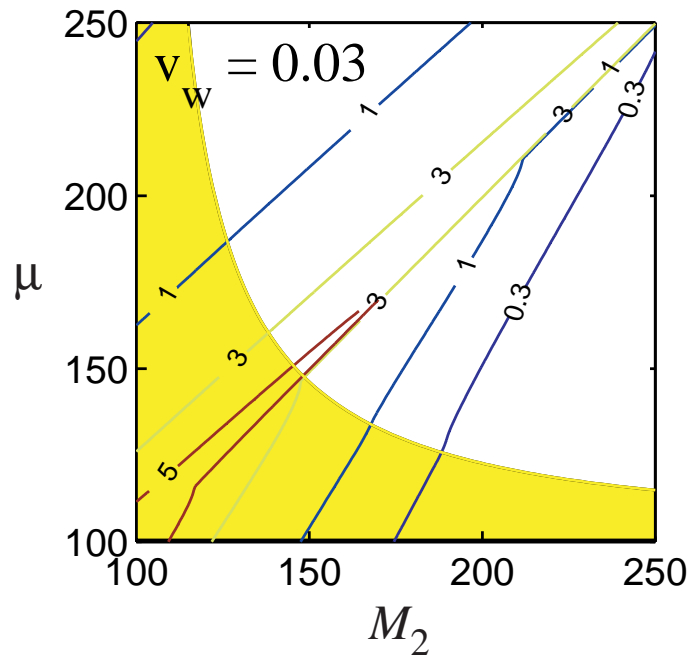

Figure 16. Constraint on the MSSM chagino parameter space in electroweak baryogenesis. 31 To generate $\eta=5 \times 10^{-10}$, the parameters must lie inside the contour labeled "5." It implies light charginos. Shaded region is excluded by LEP.

not get washed out further by the electroweak anomaly. The other is the leptogenesis, 28 where you try to generate $L \neq 0$ but no $B$ from neutrino physics well before the electroweak phase transition, and $L$ gets partially converted o $B$ due to the electroweak anomaly.

The electroweak baryogenesis is not possible in the Standard Model,29 but is still a possibility in the Minimal Supersymmetric Standard Model. However, the model is getting cornered; the available parameter space is becoming increasingly limited due to the LEP constraints on chargino, scalar top quark and Higgs boson 30 , 31 We are supposed to find a right-handed scalar top quark, charginos "soon" with a large CP violation in the chargino sector. There is possibly a detectable consequence in $B$-physics as well 32

In leptogenesis, you generate $L \neq 0$ first. Then $L$ gets partially converted to $B$ by the electroweak anomaly. The question then is how you generate $L \neq 0$. In the original proposal, 28 it was done by the decay of a 

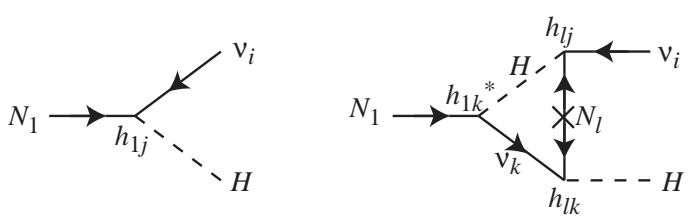

Figure 17. The tree-level and one-loop diagrams of right-handed neutrino decay into leptons and Higgs. The absorptive part in the one-loop diagram together with CP-violating phases in the Yukawa couplings leads to the direct $\mathrm{CP}$ violation $\Gamma\left(N_{1} \rightarrow l H\right) \neq$ $\Gamma\left(N_{1} \rightarrow \bar{l} H\right)$.

right-handed neutrino (say $N_{1}$ ), present in the seesaw mechanism, with a direct $\mathrm{CP}$ violation. At the tree-level, a right-handed neutrino decays equally into $l+H$ and $\bar{l}+H^{*}$. At the one-loop level, however, the interference between diagrams shown in Fig. 17 cause a difference in the decay rates of a right-handed neutrino into leptons and anti-leptons proportional to $\Im\left(h_{1 j} h_{1 k} h_{l k}^{*} h_{l j}^{*}\right)$. Much more details had been worked out in the light of recent neutrino oscillation data and it had been shown that a right-handed neutrino of about $10^{10} \mathrm{GeV}$ can well account for the cosmic baryon asymmetry from its out-ofequilibrium decay. 33 There is some tension in the supersymmetric version because of cosmological problems caused by the gravitino. But it can be circumvented for instance using the superpartner of right-handed neutrino that can have a coherent oscillation after the inflation. 34 Leptogenesis can work.

Can we prove leptogenesis experimentally? Lay Nam Chang, John Ellis, Belen Gavela, Boris Kayser, and myself got together at Snowmass and discussed this question. The short answer is unfortunately no. There are additional $\mathrm{CP}$ violating phases in the heavy right-handed neutrino sector that cannot be seen by studying the light lefthanded neutrinos. For example, even twogeneration seesaw mechanism is enough to have $\mathrm{CP}$ violation that can potentially produce lepton asymmetry, unlike the minimum of three-generations for $\mathrm{CP}$ violation in neu- trino oscillation. However, we decided that if we will see (1) electroweak baryogenesis ruled out, (2) lepton-number violation e.g. in neutrinoless double beta decay, and (3) CP violation in the neutrino sector e.g., in very longbaseline neutrino oscillation experiment, we will probably believe it based on these "archaeological" evidences.

Also, there may be additional fossils of the leptogenesis that depend on more details of the model. For instance, in the supersymmetric version with coherent right-handed sneutrino, 34 a small isocurvature component in density perturbation is created because the overall energy density and the baryon asymmetry may have independent fluctuations. If you believe in a certain scenario of supersymmetry breaking, low-energy lepton-flavor violation can carry information about CP violation in the right-handed neutrino sector. 35 Combining the above three general requirements, lepton-flavor violation and cosmological tests, the case for leptogenesis can become even stronger.

\section{Future}

Even though dramatic progress had been made, many questions remain. The important aspect of neutrino physics is that we don't stop here. It is quite a healthy field with many studies done jointly by theorists and experimentalists looking forward. Here I will discuss what is coming in the near future. 9

First of all, the oscillation signal from LSND will be verified or refuted at high confidence levels by Mini-BooNE experiment. It will start taking data in 2002. The result will mark a major branch point in the development of neutrino physics: do we need a sterile neutrino?

There is a series of experiments aimed at the oscillation signal in the atmospheric neu-

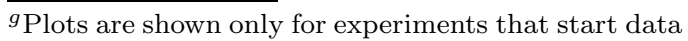
taking by the next Lepton Photon conference. 


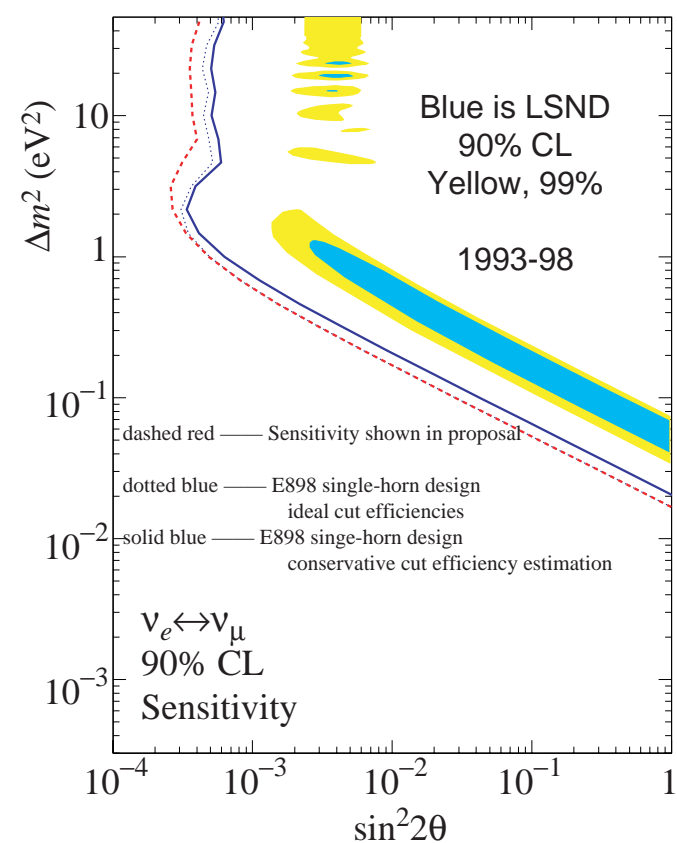

Figure 18. MiniBooNF expected $90 \%$ confidence level sensitivity limits. 36

trinos after K2K. MINOS, a long-baseline oscillation experiment from Fermilab to Sudan, Minnesota, will start in 2004, and will determine $\Delta m_{23}^{2}$ precisely. This will provide crucial input to design very long-baseline neutrino oscillation experiments of later generations. OPERA and ICARUS in Gran Sasso, using the CGNS beam from CERN, will look for $\tau$ appearance in the $\nu_{\mu}$ beam for the same $\Delta m_{23}^{2}$. All of these experiments extend reach in $\sin ^{2} 2 \theta_{13}$ as well. MONOLITH, if approved, will study atmospheric neutrinos using iron calorimetry and verify the oscillation dip.

On the solar neutrino oscillation signal, KamLAND will be the first terrestrial experiment to attack this problem. Construction has completed in 2001. Using reactor neutrinos over the baseline of about $175 \mathrm{~km}$, it will verify or exclude currently-favored LMA solution to the solar neutrino problem. If signal will be found, it will determine oscillation parameters quite well. 33 39. 20 If this turns out

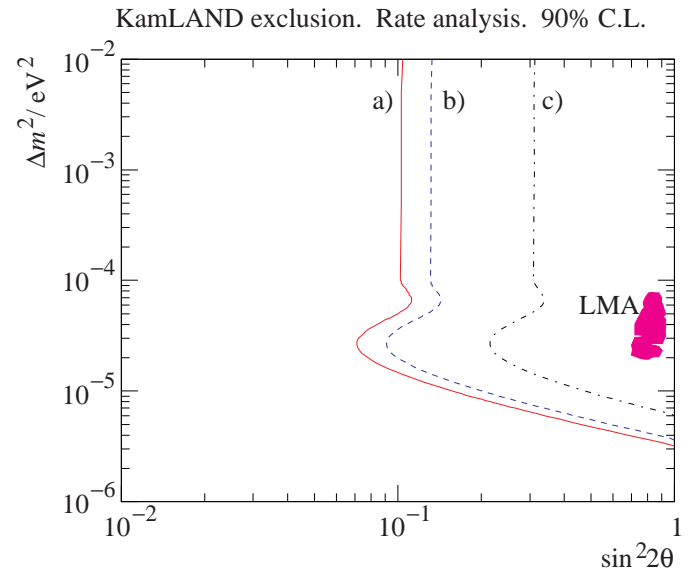

Figure 19. KamLAND expected 90\% confidence level sensitivity limits 37

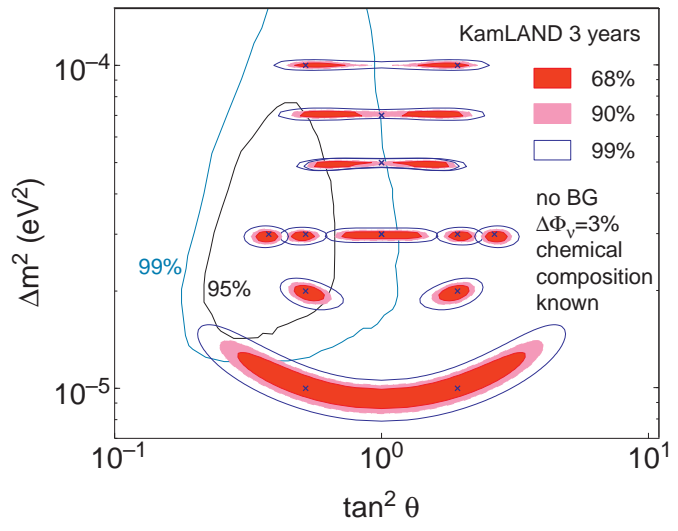

Figure 20. Mgasurement of oscillation parameters at KamLAND. 39

to be the case, it will be a dream scenario for neutrino oscillation physics. This is because $\Delta m_{\text {solar }}^{2}$ is within the sensitivity of terrestrialscale very long baseline experiments, making $\mathrm{CP}$ violation in neutrino oscillation a possible target. For example, the difference in neutrino and anti-neutrino oscillation rates is given by

$$
\begin{aligned}
& P\left(\nu_{e} \rightarrow \nu_{\mu}\right)-P\left(\bar{\nu}_{e} \rightarrow \bar{\nu}_{\mu}\right) \\
& =16 s_{12} c_{12} s_{13} c_{13}^{2} s_{23} c_{23} \sin \delta \\
& \sin \frac{\Delta m_{12}^{2}}{4 E} L \sin \frac{\Delta m_{13}^{2}}{4 E} L \sin \frac{\Delta m_{23}^{2}}{4 E} L(10)
\end{aligned}
$$

using the notation of the MNS matrix in Eq. (4). $\delta$ is the CP-violating phase. Clearly, 
$\Delta m_{12}^{2}$ has to be sizable in order for the difference not to vanish. At the same time, large $s_{13}$ is preferred. On the other hand, if LMA will be excluded, study of low-energy solar neutrinos will be crucial. KamLAND will be another major branch point.

There are already proposals to extend reach in $\sin ^{2} 2 \theta_{13}$ as well as possibly detect $\mathrm{CP}$ violation, often called neutrino superbeam experiments. One possibility is to build a neutrino beam line using $50 \mathrm{GeV}$ protons from JHF (Japan Hadron Facility) under construction, and aim the beam at SuperKamiokande. A possible upgrade of SuperKamiokande to an even bigger HyperKamiokande (?) together with more intense neutrino beam is also being discussed. On the other hand, CERN is discussing the Super Proton Linac (SPL), a GeV proton accelerator using LEP superconducting cavities. It can be aimed at a water Cherenkov detector at a modest distance to study possible $\mathrm{CP}$ violation thanks to its low energy. See Table 1 for their sensitivity.

At Snowmass, a case was made that a higher energy superbeam with a longer baseline will be beneficial. 41 For example, the matter effect can discriminate between two possible mass spectra in Fig. 田, and a longer baseline is needed for this purpose.

If KamLAND excludes the LMA solution, the study of low-energy solar neutrinos will be crucial. The predicted spectrum of solar neutrinos is shown in Fig. 21. Only realtime experiments had been done so far using ${ }^{8} \mathrm{~B}$ neutrinos.

Borexino will be the first real-time experiment to detect lower-energy solar neutrinos from the ${ }^{7} \mathrm{Be}$ line. They expect data taking starting in 2002. What is so crucial about the ${ }^{7} \mathrm{Be}$ neutrinos is that it is mono-energetic (with some broadening due to thermal collisions in the Sun). The VAC solution to the solar neutrino problem can be studied by looking for anomalous seasonal variation. The distance between the Sun and the Earth

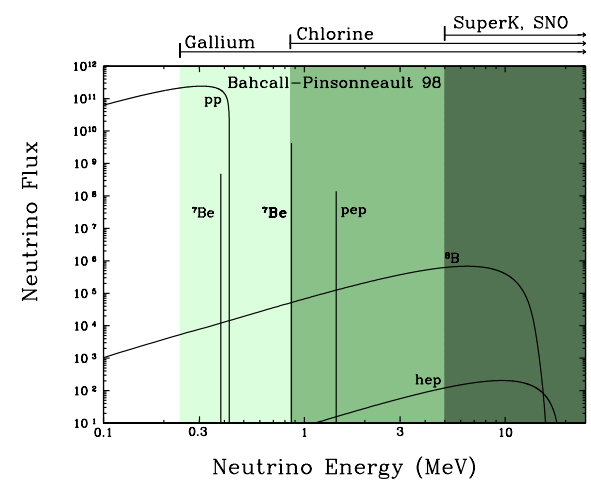

Figure 21. The spectrum of solar neutrinos 42

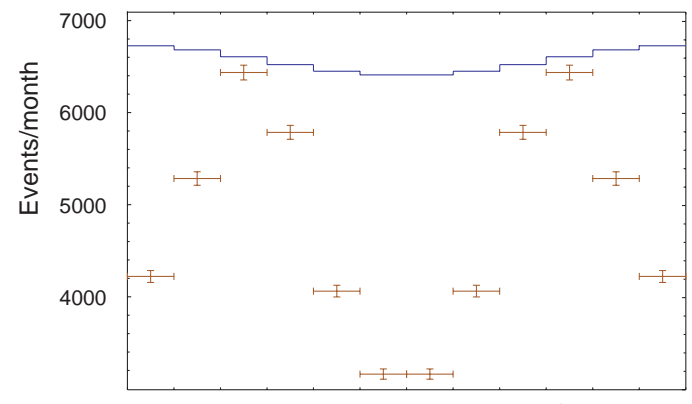

Jan Feb Mar Apr May Jun Jul Aug Sep Oct Nov Dec

Figure 22. Illustration of the effect of vacuum oscillations on the shape of the seasonal variation of the solar neutrino data. The points with statistical error bars represent the number of events/month expected at Borexino after 3 years of running for $\Delta m^{2}=3 \times 10^{-10} \mathrm{eV}^{2}, \sin ^{2} 2 \theta=1.43$

is not constant; the Sun is closer in the winter while farther in the summer in the northern hemisphere. This seasonal change in the distance causes a change in the oscillation probability for this solution and the event rate would be modulated beyond the trivial factor of $1 / r^{2}$; see Fig. 22. Borexino can look for such an anomalous seasonal variation. The sensitivity region is shown in Fig. 23. At the low $\Delta m^{2}$ region, the region is reflection symmetric. However for larger values of $\Delta m^{2}$, the region is asymmetric showing the impact of matter effect. The fact that matter effect is important even for the VAC solution had not been realized until recently. 4 This parameter 
For Publisher's use

Table 1. Physics Sensitivity for Current Superbeam Proposals 41

\begin{tabular}{llllll}
\hline \hline Name & Years of Running & kton & $\begin{array}{l}\sin ^{2} 2 \theta_{13} \text { sen- } \\
\text { sitivity }(3 \sigma)\end{array}$ & $\begin{array}{l}\text { CP Phase } \delta \\
\text { sensitivity }(3 \sigma)\end{array}$ & $\begin{array}{l}E_{\nu} \\
(\mathrm{GeV})\end{array}$ \\
\hline JHF to SuperK & 5 years $\nu$ & 50 & 0.016 & none & 0.7 \\
\hline SJHF to HyperK & 2 years $\nu, 6$ years $\bar{\nu}$ & 1000 & 0.0025 & $>15^{\circ}$ & 0.7 \\
\hline CERN to UNO & 2 years $\nu, 10$ years $\bar{\nu}$ & 400 & 0.0025 & $>40^{\circ}$ & 0.3 \\
\hline
\end{tabular}

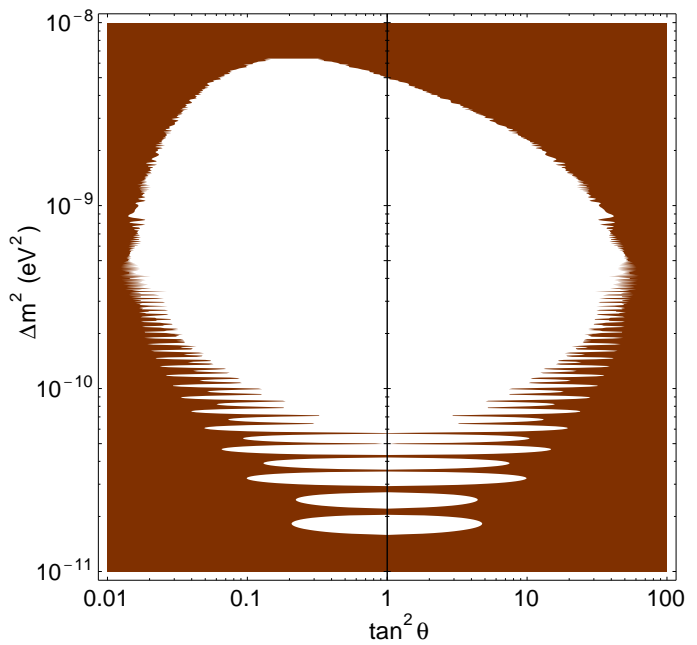

Figure 23. The parameter space Borexino is sensitive to by looking for anomalous seasonal variation in the event rate.

region was named "quasi-vacuum" 45

The LOW solution would lead to a large Earth matter effect for ${ }^{7} \mathrm{Be}$ neutrinos. 46.47 Again once found, the zenith angle dependence of the event rate depends sensitively on the oscillation parameters and can be determined quite well.

If the SMA solution turns out to be true, SNO may eventually see a distortion in the charged-current spectrum. However, the determination of parameters would require the study of $p p$ neutrinos. The point is that there is a sharp falloff in the survival parameter in the $p p$ spectrum. This is because the levelcrossing does not occur for low energy for which the matter effect does not overcome the mass splitting $\frac{\Delta m^{2}}{2 E}>\sqrt{2} G_{F} n_{e}(0)$. The location of the falloff determines $\Delta m^{2}$. On

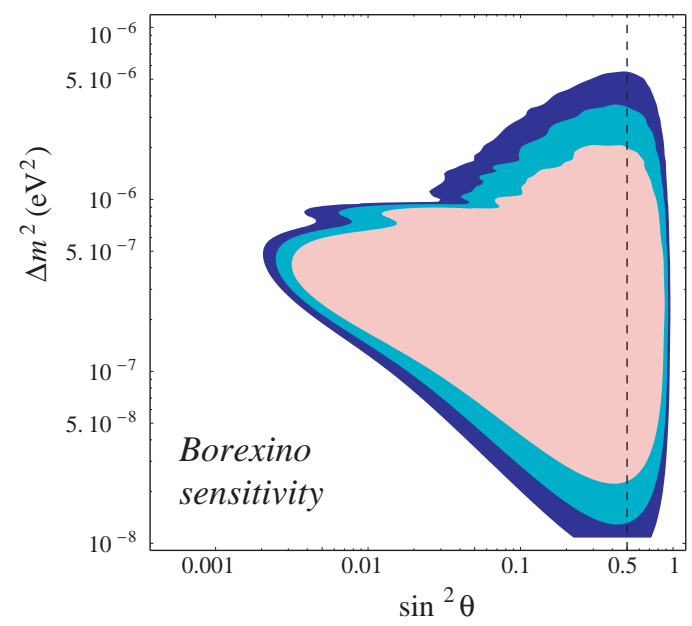

Figure $24.95 \%$ (darkest), $3 \sigma$ (dark), and $5 \sigma$ (light) sensitivity confidence level (f.L.) contours for three years of Borexino running 4 The LOW solution is completely covered.

the other hand, the slow rise at higher energy depends both on the mixing angle and the mass-squared difference.

GALLEX, SAGE, and now GNO experiments had detected $p p$ neutrinos, but they cannot measure neutrino energy spectrum and hence cannot separate $p p$ neutrinos from other solar neutrinos. There had been many proposals to study $p p$ neutrinos. Some of them use neutrino-electron elastic scattering, sensitive to both charged- and neutral-current interactions (gaseous Helium TPC, HERON with superfluid He, liquid Xe, GENIUS using solid Ge), while others use charged-current reactions on nuclei (LENS, both $\mathrm{Yb}$ and In versions, and Moon on Mo). I hope that some of them will work in the end and provide us crucial information. 
For Publisher's use
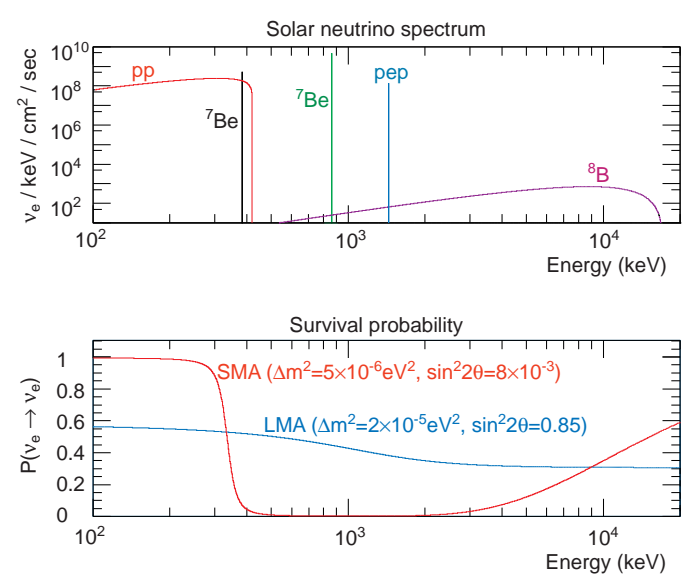

Figure 25. The survival probability as a function of neutrino energy. For SMA, there is a sharp falloff in the survival probability.

\section{Conclusions}

Neutrino physics is going through a revolution right now. We had learned a lot already, and will learn a lot more, especially on solar neutrinos. It provides an unambiguous evidence for physics beyond the minimal Standard Model.

Given strong evidences for neutrino mass, leptogenesis has gained momentum as the possible origin of cosmic baryon asymmetry. Establishing lepton-number violation would be crucial, while seeing $\mathrm{CP}$ violation in neutrino oscillation would boost the credibility. Neutrino superbeams and eventually neutrino factory could play essential role in this respect if the currently-favored LMA solution turns out to be correct.

We may not be lucky enough to test directly leptogenesis and models of neutrino masses, mixings, and flavor in general. But we can collect more "fossils" to gain insight into flavor physics at high energies, such as lepton flavor violation, combination of quark flavor physics, and even proton decay. Data may eventually point to new flavor quantum numbers that control the masses and mixings.

\section{Acknowledgments}

This work was supported in part by the DOE Contract DE-AC03-76SF00098 and in part by the NSF grant PHY-0098840.

\section{References}

1. Jordan Goodman, in this proceedings.

2. Josh Klein, in this proceedings.

3. Chang-Kee Jung, in this proceedings.

4. Shigeki Aoki, in this proceedings.

5. G. L. Fogli, E. Lisi and D. Montanino, Phys. Rev. D 54, 2048 (1996) arXiv:hep-ph/9605273.

6. A. de Gouvêa, A. Friedland and H. Murayama, Phys. Lett. B 490, 125 (2000) arXiv:hep-ph/0002064.

7. J. N. Bahcall, M. C. Gonzalez-Garcia and C. Peña-Garay, JHEP 0108, 014 (2001) arXiv:hep-ph/0106258. See also G. L. Fogli, E. Lisi, D. Montanino and A. Palazzo, Phys. Rev. D 64, 093007 (2001) arXiv:hep-ph/0106247; A. Bandyopadhyay, S. Choubey, S. Goswami and K. Kar, Phys. Lett. B 519, 83 (2001) arXiv:hep-ph/0106264; P. I. Krastev and A. Y. Smirnov, arXiv:hepph/0108177. P. Aliani, V. Antonelli, M. Picariello and E. Torrente-Lujan, arXiv:hep-ph/0111418.

8. J. N. Bahcall, M. H. Pinsonneault and S. Basu, Astrophys. J. 555, 990 (2001) arXiv:astro-ph/0010346.

9. For the most recent analysis with four-flavors including a sterile neutrino, see M. C. Gonzalez-Garcia, M. Maltoni and C. Peña-Garay, arXiv:hepph/0108073. See also V. D. Barger, D. Marfatia and K. Whisnant, Phys. Rev. Lett. 88, 011302 (2002) arXiv:hep$\mathrm{ph} / 0106207$, and a paper that appeared after the conference A. Strumia, arXiv:hep-ph/0201134.

10. M. Apollonio et al. [CHOOZ Collabo- 
ration], Phys. Lett. B 466, 415 (1999) arXiv:hep-ex/9907037; F. Boehm et al., Phys. Rev. D 64, 112001 (2001) arXiv:hep-ex/0107009.

11. S. M. Bilenkii, C. Giunti, W. Grimus and T. Schwetz, Phys. Rev. D 60, 073007 (1999) arXiv:hep-ph/9903454;

12. V. D. Barger, B. Kayser, J. Learned, T. Weiler and K. Whisnant, Phys. Lett. B 489, 345 (2000) arXiv:hep$\mathrm{ph} / 0008019$.

13. M. Maltoni, T. Schwetz and J. W. Valle, Phys. Lett. B 518, 252 (2001) arXiv:hep-ph/0107150.

14. H. Murayama and T. Yanagida, Phys. Lett. B 520, 263 (2001) arXiv:hep$\mathrm{ph} / 0010178$.

15. T. Yanagida, "Horizontal Symmetry And Masses Of Neutrinos", Prog. Theor. Phys. 64 (1980) 1103, and in Proceedings of the "Workshop on the Unified Theory and the Baryon Number in the Universe", Tsukuba, Japan, Feb 13-14, 1979, Eds. O. Sawada and A. Sugamoto, KEK report KEK-79-18, p. 95; M. Gell-Mann, P. Ramond and R. Slansky, in "Supergravity" (North-Holland, Amsterdam, 1979) eds. D.Z. Freedman and P. van Nieuwenhuizen, Print80-0576 (CERN).

16. K. R. Dienes, E. Dudas and T. Gherghetta, Nucl. Phys. B 557, 25 (1999) arXiv:hepph/9811428]; N. Arkani-Hamed, S. Dimopoulos, G. R. Dvali and J. MarchRussell, talk presented at SUSY 98 Conference, Oxford, England, 11-17 Jul 1998. arXiv:hep-ph/9811448; Y. Grossman and M. Neubert, Phys. Lett. B 474, 361 (2000) arXiv:hep-ph/9912408].

17. L. J. Hall, H. Murayama and N. Weiner, Phys. Rev. Lett. 84, 2572 (2000) arXiv:hep-ph/9911341.

18. N. Haba and H. Murayama, Phys. Rev. D 63, 053010 (2001) arXiv:hep$\mathrm{ph} / 0009174$.
19. Riccardo Barbieri, in this proceedings.

20. For a review, see H. Murayama, lectures at TASI 2000, http://hitoshi. berkeley.edu/TASI/

21. John Ellis, in this proceedings.

22. M. Yoshimura, Phys. Rev. Lett. 41, 281 (1978) [Erratum-ibid. 42, 746 (1978)]; A. Y. Ignatev, N. V. Krasnikov, V. A. Kuzmin and A. N. Tavkhelidze, Phys. Lett. B 76, 436 (1978).

23. R. Kessler, in this proceedings.

24. L. Iconomidou-Fayard, in this proceedings.

25. G. 't Hooft, Phys. Rev. Lett. 37, 8 (1976).

26. V. A. Kuzmin, V. A. Rubakov and M. E. Shaposhnikov, Phys. Lett. B 155, 36 (1985).

27. S. Y. Khlebnikov and M. E. Shaposhnikov, Nucl. Phys. B308 (1988) 885;

J. A. Harvey and M. S. Turner, Number Violation," Phys. Rev. D 42 (1990) 3344.

28. M. Fukugita and T. Yanagida, Phys. Lett. B 174, 45 (1986).

29. See a review and references therein: A. G. Cohen, D. B. Kaplan and A. E. Nelson, Ann. Rev. Nucl. Part. Sci. 43, 27 (1993) arXiv:hep-ph/9302210.

30. M. Carena, J. M. Moreno, M. Quiros, M. Seco and C. E. Wagner, Nucl. Phys. B 599, 158 (2001) arXiv:hep$\mathrm{ph} / 0011055$.

31. J. M. Cline, M. Joyce and K. Kainulainen, JHEP

0007 , 018 (2000) arXiv:hep-ph/0006119; Erratum, arXiv:hep-ph/0110031.

32. H. Murayama and A. Pierce, in preparation.

33. See, e.g., W. Buchmuller, Presented at 8th International Symposium on Particle Strings and Cosmology (PASCOS 2001), Chapel Hill, North Carolina, 10-15 Apr 2001, arXiv:hep-ph/0107153, and references therein.

34. K. Hamaguchi, H. Murayama and 
T. Yanagida, arXiv:hep-ph/0109030.

35. J. R. Ellis, J. Hisano, S. Lola and M. Raidal, arXiv:hep-ph/0109125.

36. A. Bazarko [MiniBooNE Collaboration], talk presented at 19th International Conference on Neutrino Physics and Astrophysics - Neutrino 2000, Sudbury, Ontario, Canada, 16-21 Jun 2000. Published in proceedings, Nucl. Phys. Proc. Suppl. 91, 210 (2000) arXiv:hep$\mathrm{ex} / 0009056$.

37. J. Busenitz et al., "Proposal for US Participation in KamLAND," March 1999, http://kamland.lbl.gov/.

38. V. D. Barger, D. Marfatia and B. P. Wood, Phys. Lett. B 498, 53 (2001) arXiv:hep-ph/0011251.

39. H. Murayama and A. Pierce, Phys. Rev. D 65, 013012 (2002) arXiv:hep$\mathrm{ph} / 0012075$.

40. A. de Gouvêa and C. Peña-Garay, Phys. Rev. D 64, 113011 (2001) arXiv:hep$\mathrm{ph} / 0107186$.

41. "E1 working group summary: Neutrino factories and muon colliders," in Proc. of the APS/DPF/DPB Summer Study on the Future of Particle Physics (Snowmass 2001) ed. R. Davidson and C. Quigg, arXiv:hep-ph/0111030.

42. Taken from http://www.sns.ias.edu/ jnb/SNviewgraphs/.

43. A. de Gouvêa, A. Friedland and H. Murayama, Phys. Rev. D 60, 093011 (1999) arXiv:hep-ph/9904399.

44. A. Friedland, Phys. Rev. Lett. 85, 936 (2000) arXiv:hep-ph/0002063.

45. G. L. Fogli, E. Lisi, D. Montanino and A. Palazzo, Phys. Rev. D 62, 113004 (2000) arXiv:hep-ph/0005261.

46. A. de Gouvêa, A. Friedland and H. Murayama, JHEP 0103, 009 (2001) arXiv:hep-ph/9910286.

47. G. L. Fogli, E. Lisi, D. Montanino and A. Palazzo, Phys. Rev. D 61, 073009 (2000) arXiv:hep-ph/9910387. 